ing. Andreas von Mach

General Manager, vMACH Engineering, GERMANY

dr ing. Pawet Pawlewski

Poznan University of Technology, POLAND

\title{
New concepts for test and FE analysis data comparisons
}

The paper proposes usage of existing methods of test data analysis into post-processing of FE models results, as well as usage of improved methods of FE data identifications for test data analysis. Continuously growing size and complexity of the FE models and possibilities of new technologies like laser or camera measurement of vibrations need new methods in identification, comparison and correlation. Generalised workflow of NVH analysis and 18 steps of proposed methodology are presented. Every step is described using graphics pictures generated from FEgraph software dedicated as post-processing tool for automotive industry. The proposed methods of same data processing for test and analysis data was successfully introduced into automotive industry and is on continuous development. Nevertheless, it can be used in the other branches of industry including in the analysis of the strength calculations of the rail vehicles bodies.

\section{Introduction}

Large FE-Models are characterized by: size growing continuously (over $1 \mathrm{M}$ DOFs), many variants, many load cases (sub-cases), different materials (100s in one model), different joints (10000s), FE-meshing done by suppliers. Test data are formed using new measure technologies - laser, cameras. The paper proposes usage of existing methods of test data analysis into post-processing of FE models results, as well as usage of improved methods of FE data identifications for test data analysis. Figure 1. presents generalised workflow of NVH analysis. Proposed methodology is described in chapter 2. Steps of this methodology are described using many graphics pictures generated from FEgraph software dedicated as post-processing tool in automotive industry.

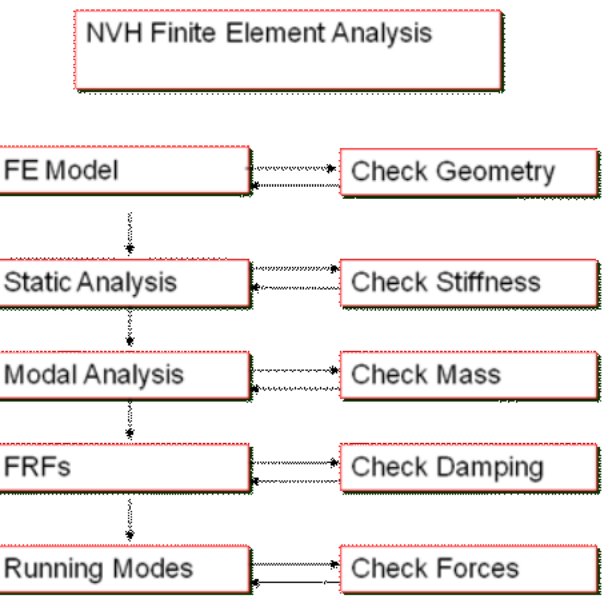

\section{Methodology}

Proposed methodology is composed of 18 steps:

1. Data processing

2. Geometry Correlation

3. Stability Diagrams

4. Auto MAC

5. Auto MAC Optimization

6. Damping Identification

7. Complexity

8. Pre - Testing

9. MAC

10. CoMAC

11. Participation Factors

12. Symmetric and Anti-Symmetric Components

Figure 1: Generalised workflow of NVH analysis. 

13. Form Comparison
16. FRF Comparison
14. Total Energy Analysis
17. Model Updating
15. SUMFactor
18. FRF Updating

STEP - 1. Geometry Correlation (based on test and FE model geometry)
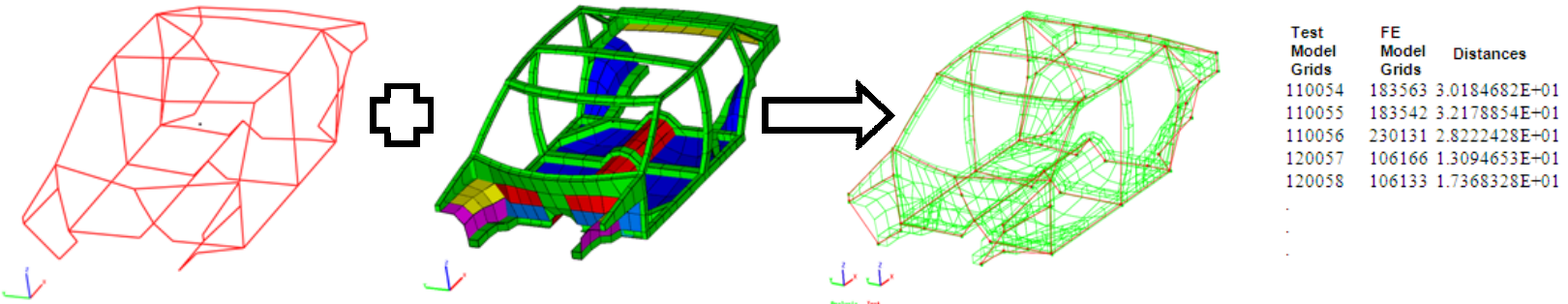

Figure 2: Geometry correlation of test and FE models. (results are as node map)

STEP - 2. Stability Diagrams (based on measured or calculated Frequency Response and grids) Applied methods are: ERA, LSCE, LSCF.
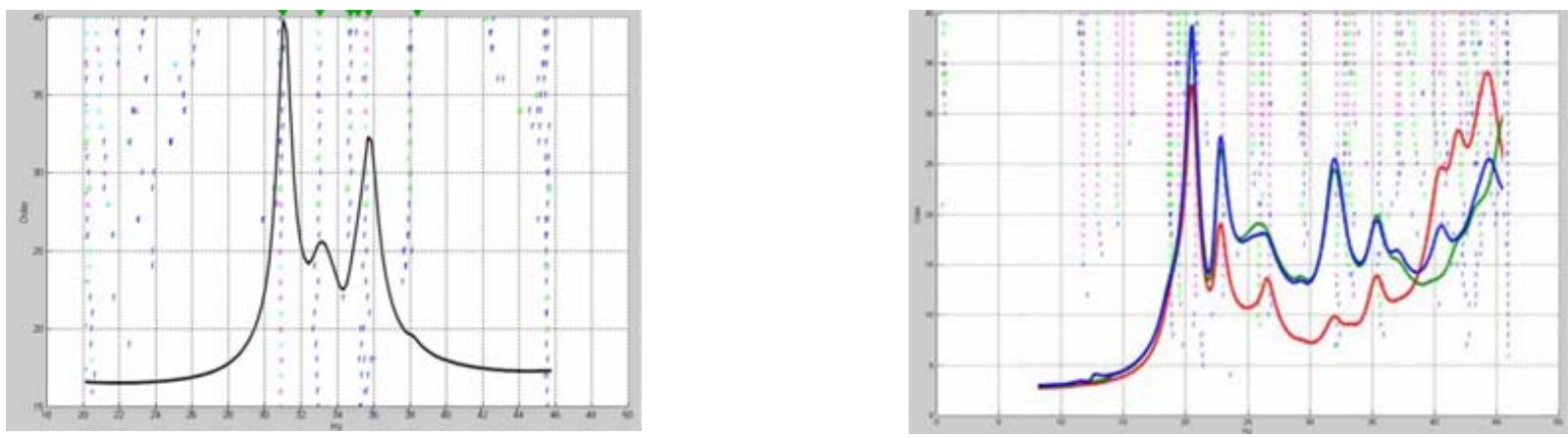

Figure 3: Stabilisation diagrams.
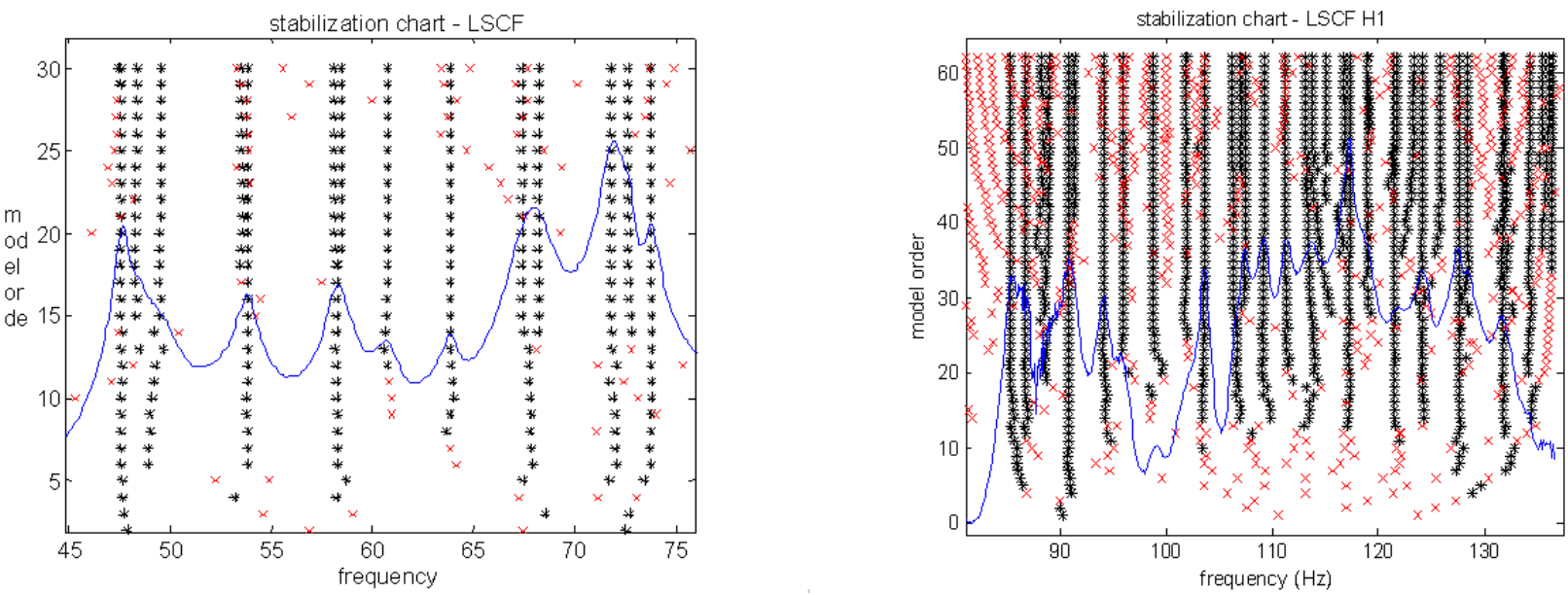

Figure 4: $\quad$ Stabilisation charts - LSCF method based on TechPro Technology.

\begin{tabular}{|c|c|c|c|c|c|c|c|}
\hline \multirow{2}{*}{\multicolumn{2}{|c|}{ LSCE }} & & & & & \multicolumn{2}{|c|}{ Analysis } \\
\hline & & \multicolumn{2}{|c|}{ ERA } & \multicolumn{2}{|c|}{ LSCF } & Mode & MAC \\
\hline $\mathrm{Hz}$ & $\%$ & $\mathrm{~Hz}$ & $\%$ & $\mathrm{~Hz}$ & $\%$ & $\mathrm{~Hz}$ & \\
\hline 67.47 & 1.08 & 67.48 & 1.06 & 67.40 & 9,92 & 67.07 & 0.810 \\
\hline & & 67.69 & 2.91 & & & & \\
\hline 68.13 & 1.19 & 68.13 & 1.18 & 68.25 & 1.14 & 69.60 & 0.463 \\
\hline 71.84 & 0.65 & 71.83 & 0.65 & 71.82 & 0.66 & 71.61 & 0.511 \\
\hline 72.65 & 0.51 & 72.65 & 0.51 & 72.66 & 0.47 & 73.80 & 0.651 \\
\hline
\end{tabular}

Figure 5: $\quad$ Results table - LSCE, ERA, LSCF methods . 
STEP - 3. Auto MAC (Modal Assurance Criterion - based on test or FE model mode shapes)
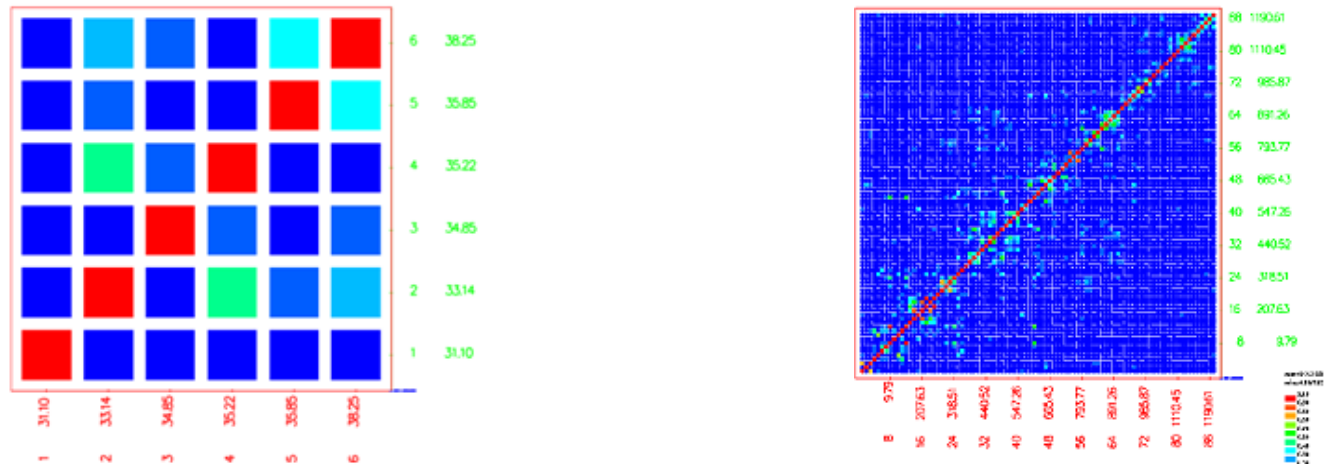

Figure 6: Auto MAC graphics presentation - test and FE model mode shapes.

STEP - 4. Auto MAC Optimization

Before

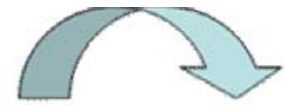

After

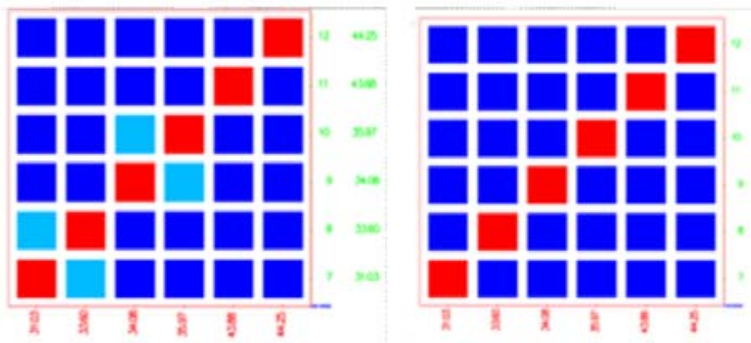

Figure 7: Results of Auto MAC optimization process.

STEP - 5. Damping Identification (based on measured or calculated Frequency Response, using Circle Fitting Method)

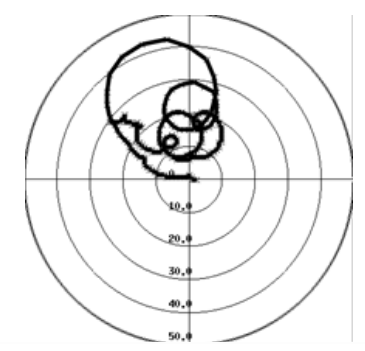

$$
\xi_{r}=\frac{\omega_{2}-\omega_{1}}{\omega_{r \cdot}\left[\operatorname{tg}\left(\frac{\theta_{1}}{2}\right)+\operatorname{tg}\left(\frac{\theta_{2}}{2}\right)\right]}
$$
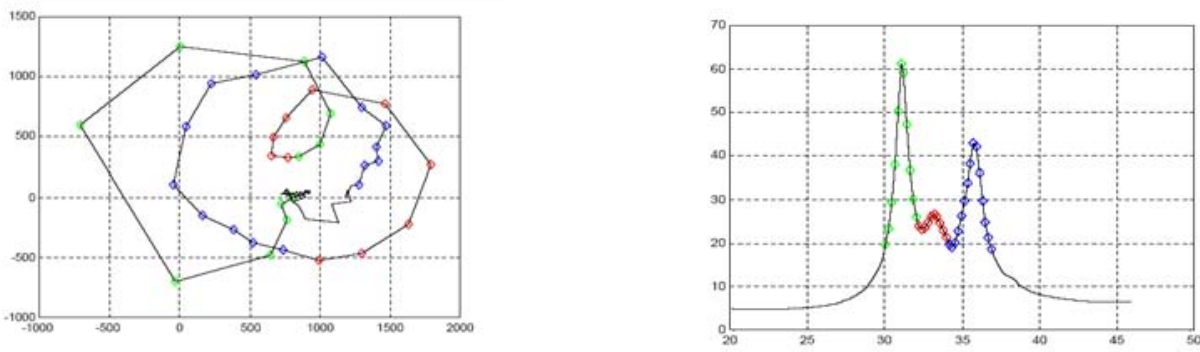

Figure 8: Circle Fitting methods for damping identification.
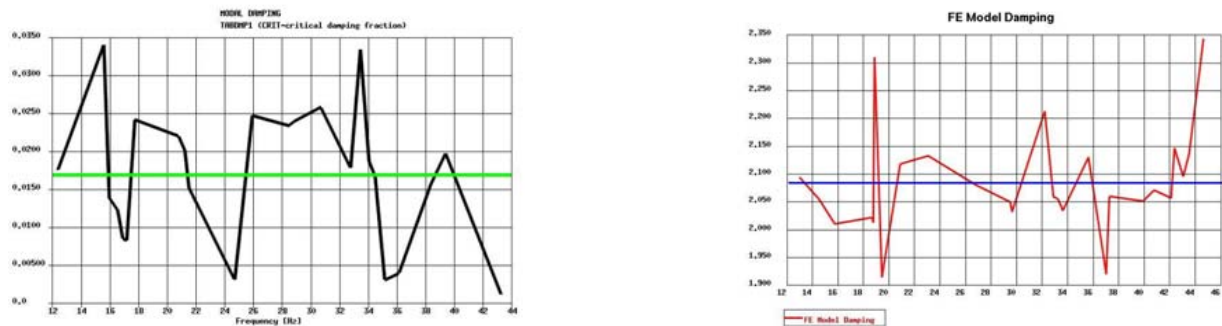

Figure 9: Modal damping results from test and FE analysis (green and blue lines sign mean values). 
STEP - 6. Complexity (based on Complex Mode Shapes and Geometry following things are calculated: Modal Phase Co-linearity, Mean Phase, Deviation Mean Phase)
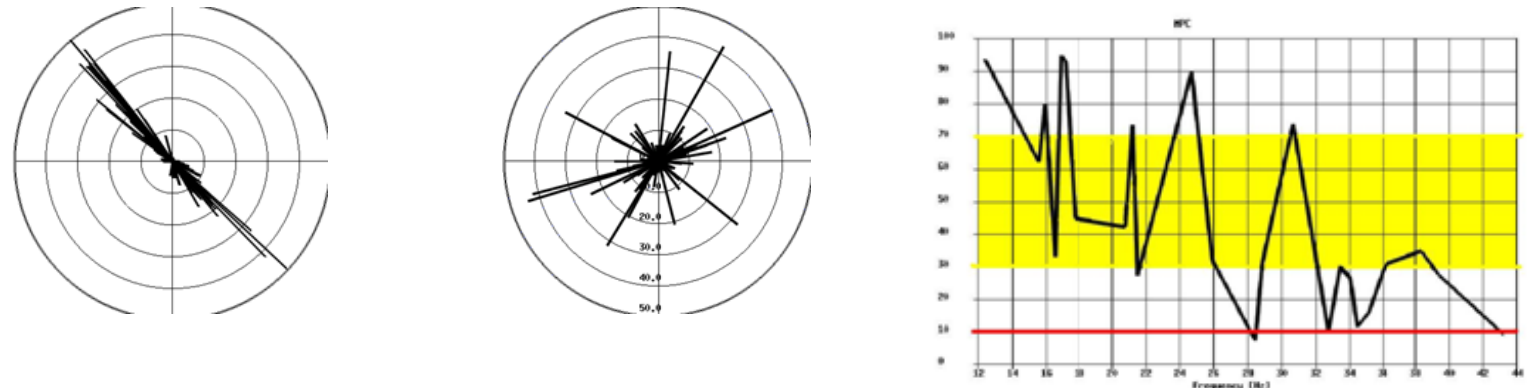

Figure 10: $\quad$ Results from Complexity step. (for MPC - results above yellow area are correct, in yellow are are questionable and below are incorrect).

STEP - 7. Pre - Testing (based on FE model geometry and Mode Shapes, using following Pre-Testing Methods: Optimum Driving Point (ODP), Non-Optimum Driving Point (NODP), Average Driving DOF Displacement (ADDOF-D), Average Driving DOF Velocity (ADDOF-V), Average Driving DOF Acceleration (ADDOF-A), Effective Independence (EI))

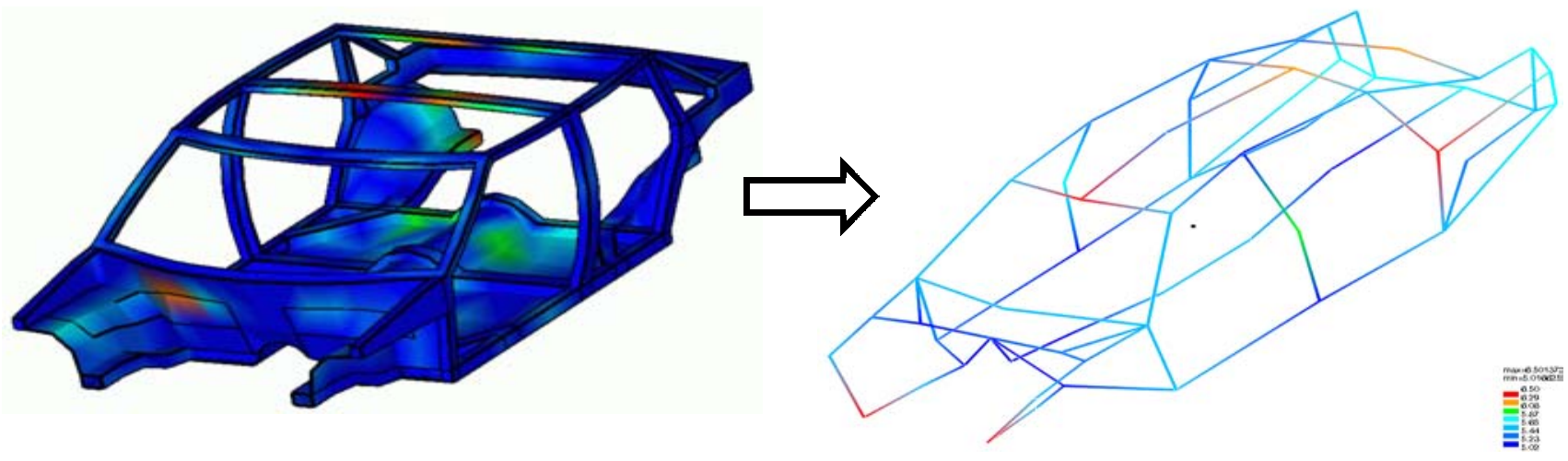

Figure 11: Pre-Testing results - reduced an converted FE model into a test model with selected measure points for modal analysis.

STEP - 8. Modal Assurance Criterion -MAC (based on test and FE model mode shapes and node map)
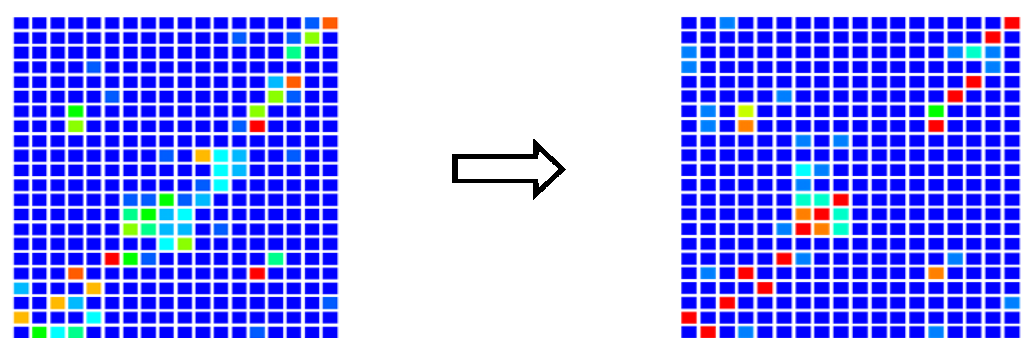

Figure 12: Tranformation from reduced test model into expanded test model .

STEP - 9. Coordinate Modal Assurance Criterion - CoMAC (based on test and FE model mode shapes, node map and mode pairs).

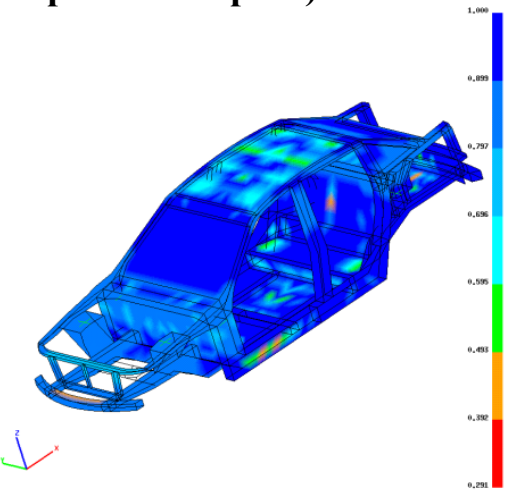

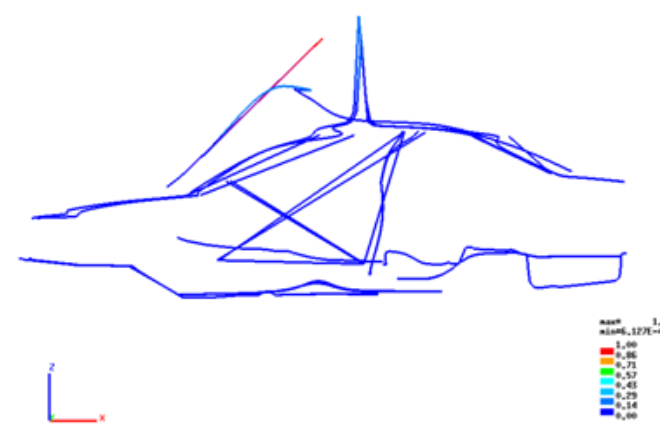

Figure 13: Graphics presentation of local differences between two compared models. 


\section{STEP - 10. Participation Factors}
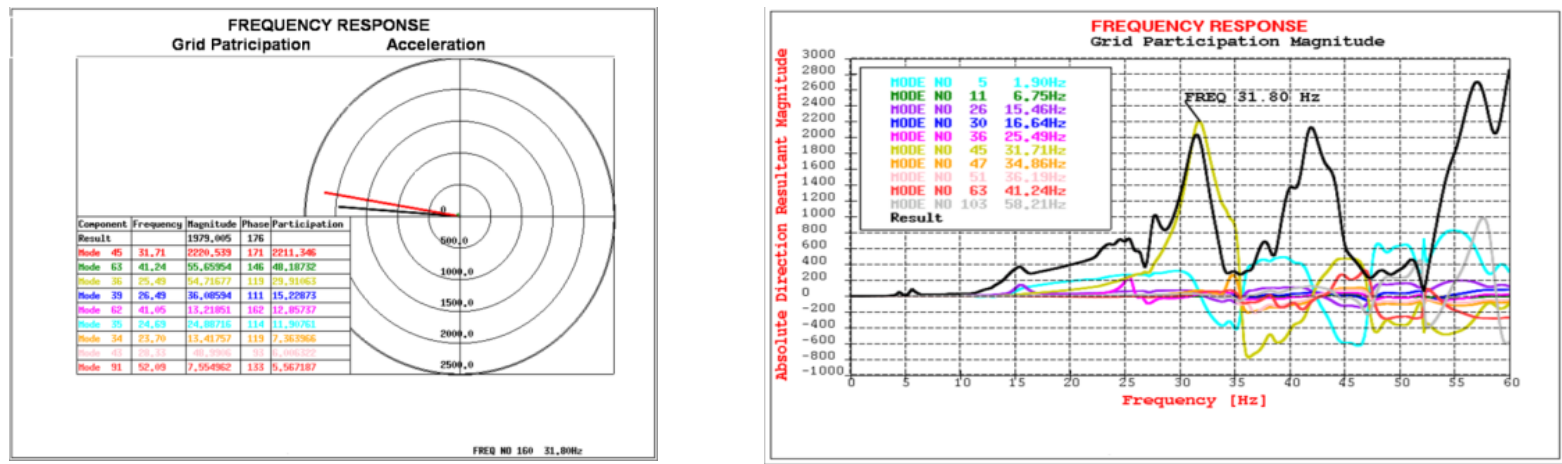

Figure 14: Participation of DOF Responses (Grid participation - acceleration polar diagram and amplitude XY chart)
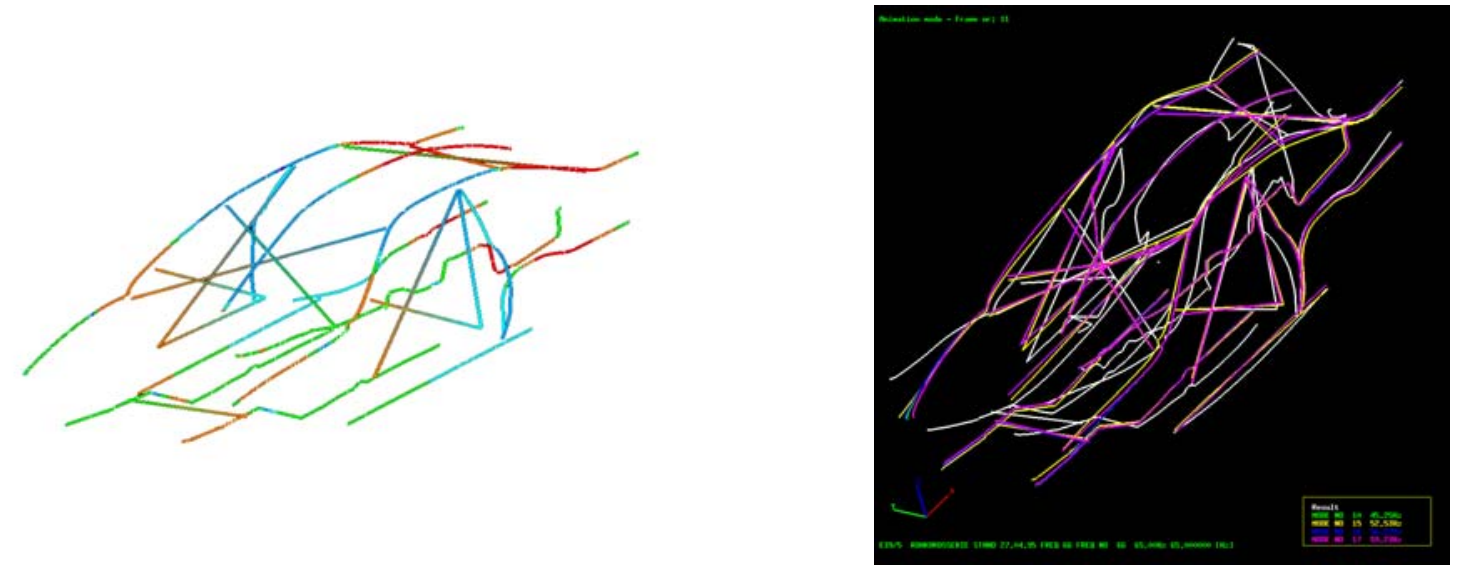

Figure 15: Participation of modes 3D.

STEP - 11. Symmetric and Anti-symmetric Components (based on mode shapes and geometry).
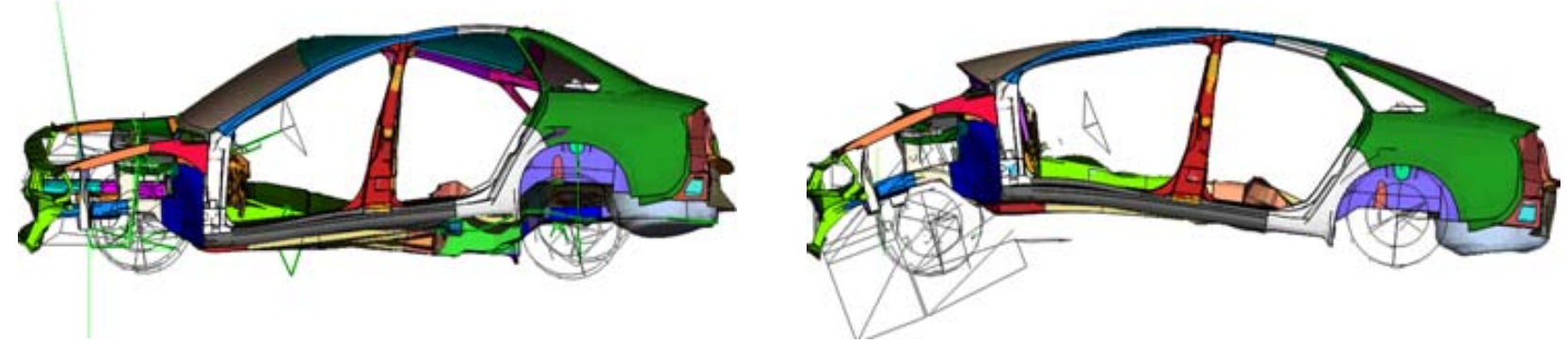

Figure 16: Symmetric (58.55\%) and anti-symmetric (41.45\%) components .

\begin{tabular}{|c|c|c|c|}
\hline \multicolumn{2}{|c|}{} & Symmetric & Asymmetric \\
\hline \multirow{3}{*}{ Translation } & T1 & + & - \\
\cline { 2 - 4 } & T2 & - & + \\
\cline { 2 - 4 } & T3 & + & - \\
\hline \multirow{3}{*}{ Rotation } & R1 & - & + \\
\cline { 2 - 4 } & R2 & + & - \\
\cline { 2 - 4 } & R3 & - & + \\
\hline
\end{tabular}

Figure 17: Table with rules for formation symmetric and anti-symmetric components. 

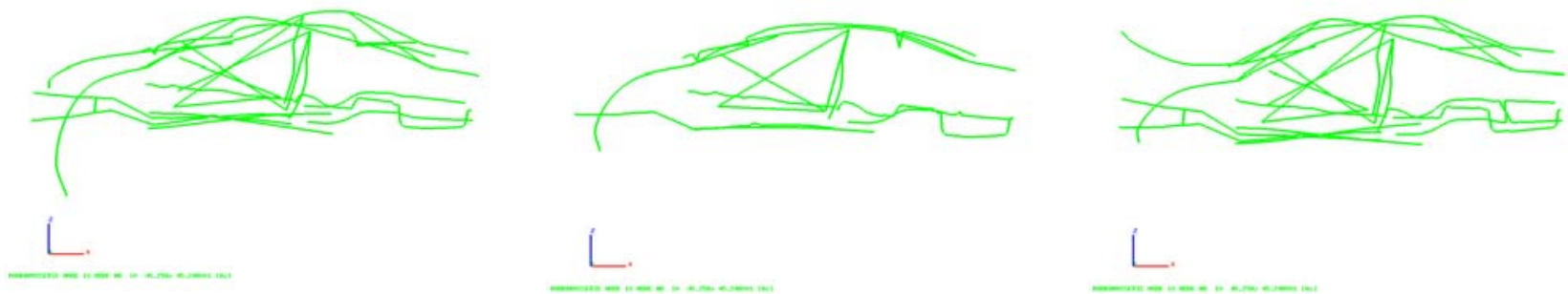

Figure 18: Complete model, symmetric and antisymmetric components .

STEP - 12. Form Comparison (based on mode shapes, geometry, node map).
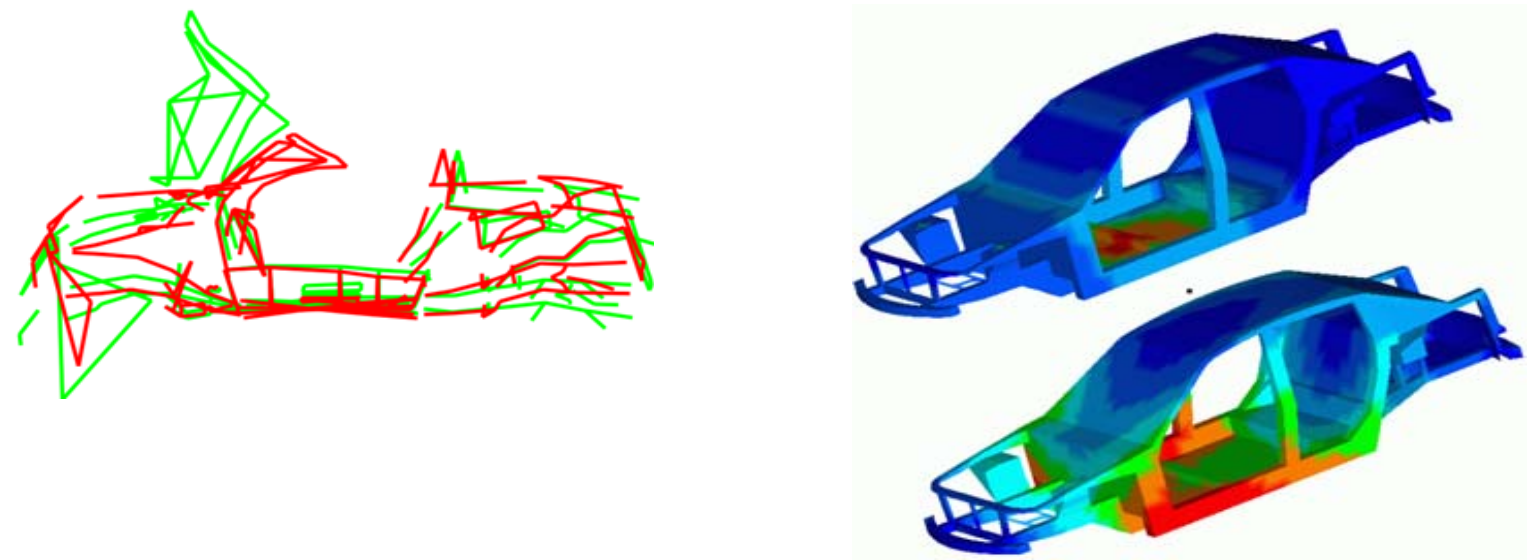

Figure 19: Comparison frames for two models, chosen modes pair.

STEP - 13. Total Energy Analysis (based on geometric interpretation of complex modal energy, Frequency Response, mode shapes and geometry)
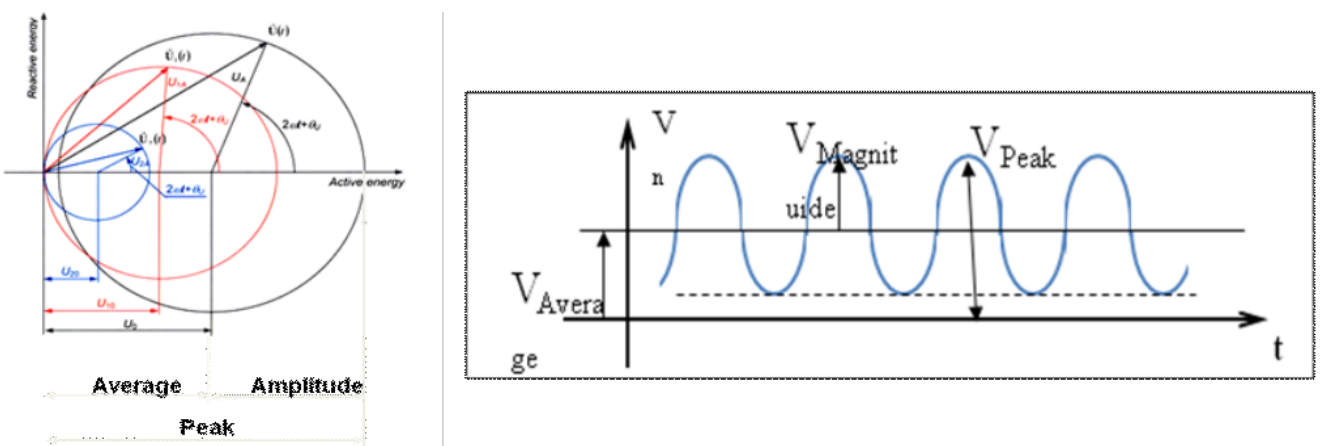

Figure 20: Geometric interpretation of complex modal energy.
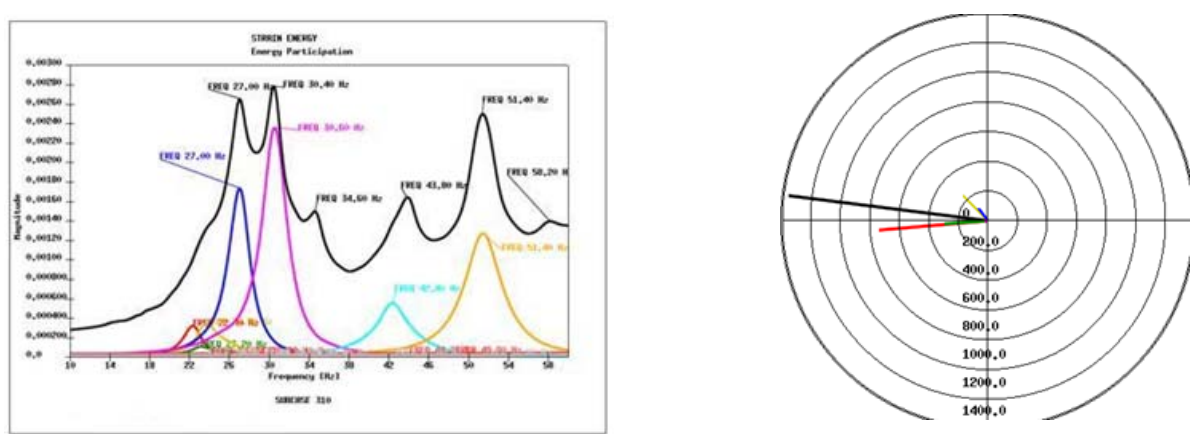

Figure 21: Results of total energy analysis - identification of dominant modes. 
STEP - 14. SUM Factor (based on test or/and FE model Frequency Response)
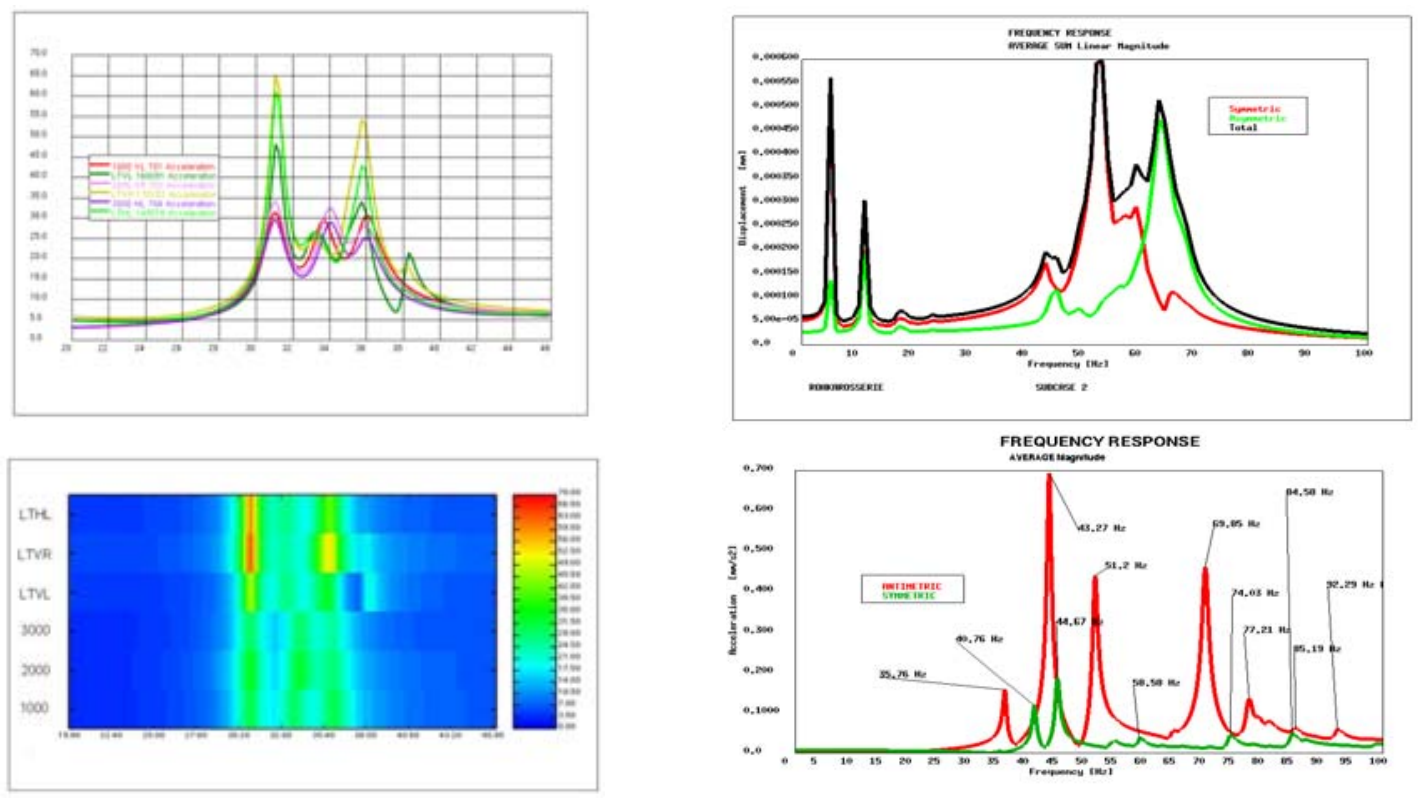

Figure 22: Results of SUM Factor step - identification of global and dominant modes.

STEP - 15. FRF Comparison (based on Frequency Response, geometry and node map using: Frequency Response - FR, Frequency Response Assurance Criterion - FRAC, Frequency Response Scale Factor FRSF, Frequency Domain Assurance Criterion - FDAC, Response Vector Assurance Criterion - RVAC, Modal Frequency Assurance Criterion - MFAC).
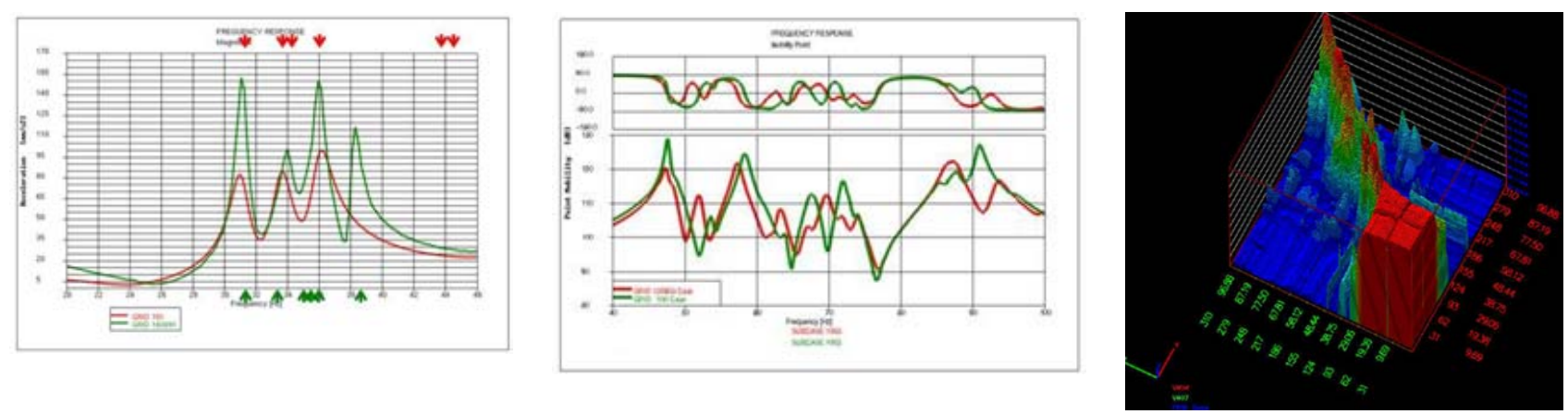

Figure 23: Results of FRF comparison (part 1).
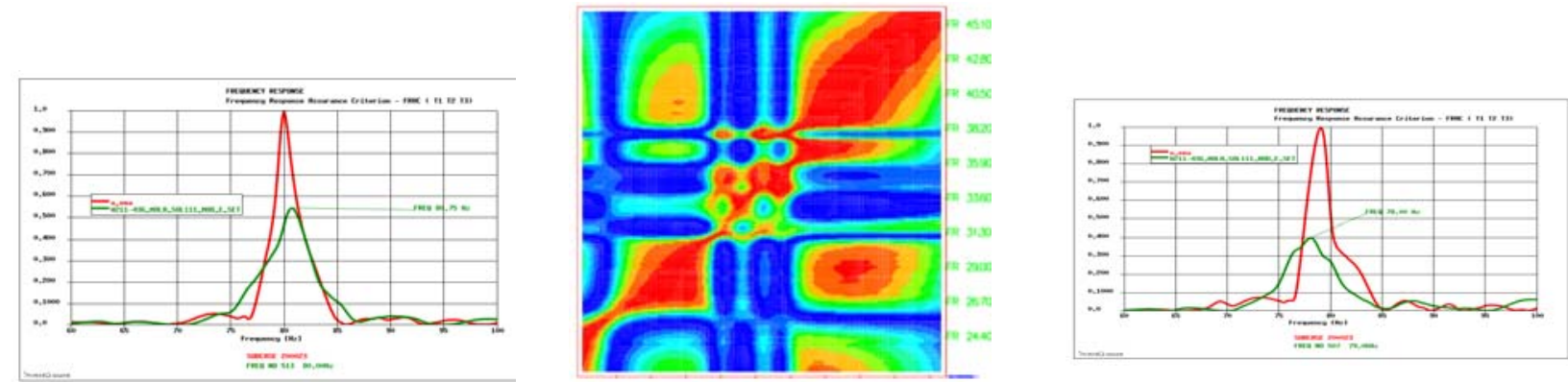

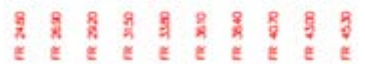

Figure 24: Results of FRF comparison (part 2). 
STEP - 16. Coordinate FRF's (Amplitude Difference, Phase Difference, Error Variations, Coordinate Frequency Response Assurance Criterion - CoFRAC, Improved Coordinate Frequency Response Assurance Criterion - ICoFRAC).

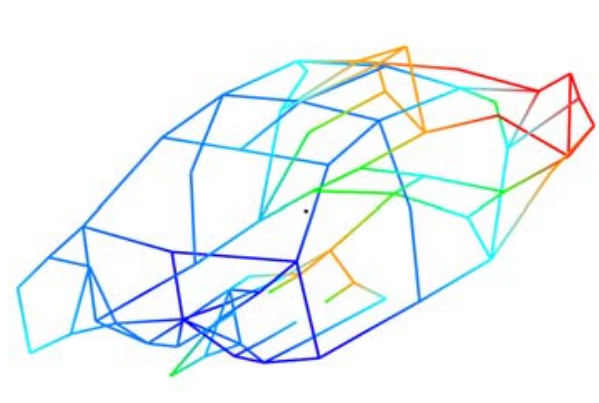

\begin{tabular}{|c|c|c|}
\hline & Normal Modes & FRF \\
\hline Amplitude & -1 or 1 & $\begin{array}{c}-180^{\circ} \text { to } \\
180^{\circ}\end{array}$ \\
\hline Phase & - & 0 to 1 \\
\hline FRAC & - & \\
\hline FRFSF & - & 0 to 1 \\
\hline FDAC & - & -1 to 1 \\
\hline iFDAC & - &
\end{tabular}

Figure 25: Coordinate FRF's results (part 1).

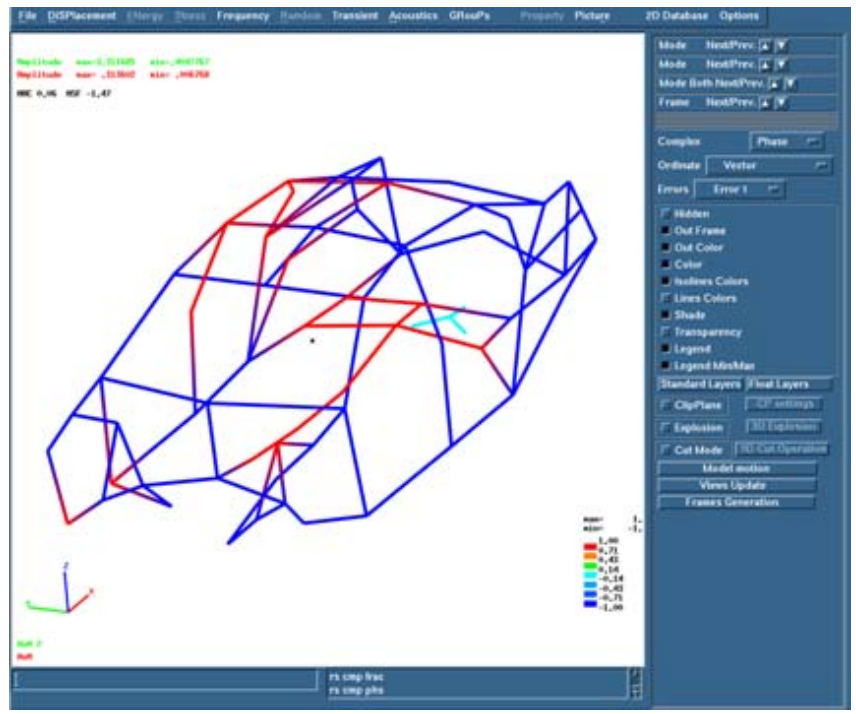

\begin{tabular}{|c|c|}
\hline Normal Modes & FRF \\
\hline MAC & FRAC \\
\hline MSF & FRSF \\
\hline
\end{tabular}

Figure 26: Coordinate FRF's results (part 2).

STEP - 17. Model Updating (based on methods: directly matrix FE-Output, Energy and force based recalculation (Kinetic, Strain, Dissipation), program direct calculation, approximated \& simplified)
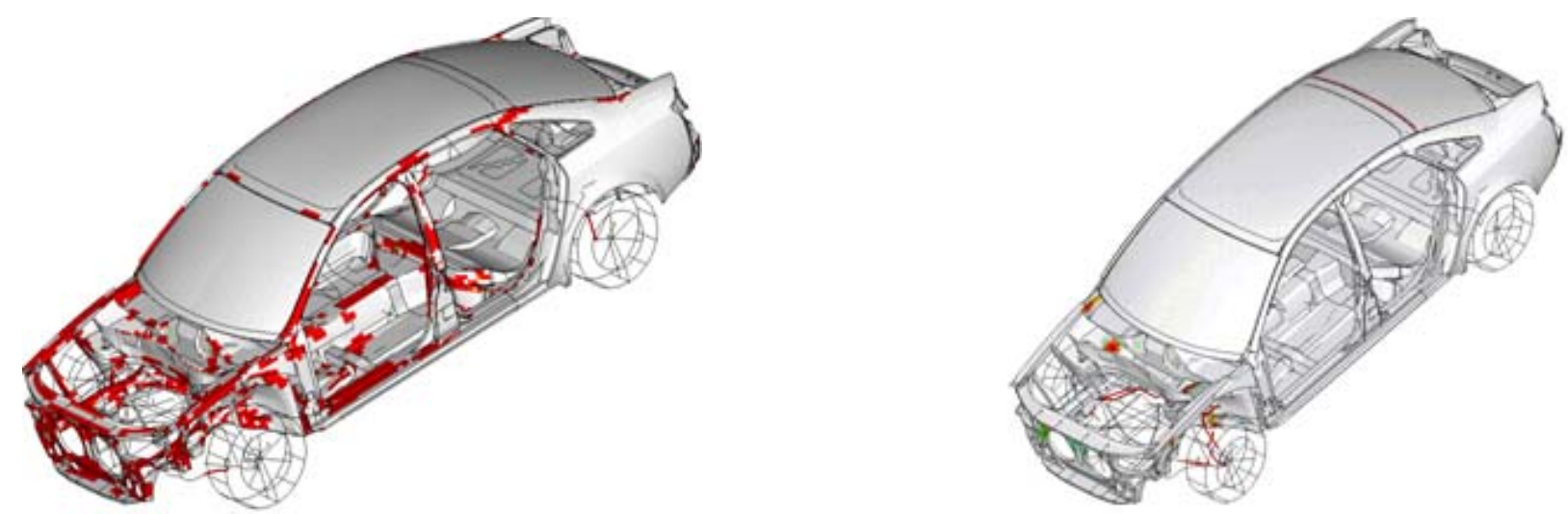

Figure 27: Eigenvalues and eigenvectors sensitivity values. 


\begin{tabular}{|l|c|}
\hline Method & Equation \\
\hline SUM - Factor & $A=\sum \frac{1}{3 n} \sum\left(\left|U_{i}\right|+\left|V_{i}\right|+\left|W_{i}\right|\right)$ \\
\hline $\begin{array}{l}\text { FRF (Frequency Response Function) } \\
\text { Only Magnitude will by keep count }\end{array}$ & $\left.\Delta F R F=\mid H_{X i j}(\omega)\right\}|-|\left\{H_{A i j}(\omega)\right\} \mid$ \\
\hline $\begin{array}{l}\text { LS- Error (Frequency Response Function) } \\
\text { Only Magnitude will by keep count }\end{array}$ & $F R L S=\frac{\left|\left\{H_{X i j}(\omega)-H_{A i j}(\omega)\right\}^{H}\left\{H_{X i j}(\omega)-H_{A i j}(\omega)\right\}\right|}{\left(\left\{H_{X i j}(\omega)\right\}^{H}\left\{H_{X i j}(\omega)\right\}\right)}$ \\
\hline $\begin{array}{l}\text { FRAC (Frequency Response Assurance } \\
\text { Criterion) Magnitude and Phase will by } \\
\text { keep count }\end{array}$ & $F R A C=\frac{\left|\left\{H_{X i j}(\omega)\right\}^{H}\left\{H_{A i j}(\omega)\right\}\right|^{2}}{\left(\left\{H_{X i j}(\omega)\right\}^{H}\left\{H_{X i j}(\omega)\right\}\right)\left(\left\{H_{A i j}(\omega)\right\}^{H}\left\{H_{A i j}(\omega)\right\}\right)}$ \\
\hline $\begin{array}{l}\text { FRSF (Frequency Response Scale Factor) } \\
\text { Evaluates mmagnitude }\end{array}$ & $F R S F=\frac{2\left|\left\{H_{X i j}(\omega)\right\}^{H}\left\{H_{A i j}(\omega)\right\}\right|}{\left(\left\{H_{X i j}(\omega)\right\}^{H}\left\{H_{X i j}(\omega)\right\}\right)+\left(\left\{H_{A i j}(\omega)\right\}^{H}\left\{H_{A i j}(\omega)\right\}\right)}$ \\
\hline
\end{tabular}

Figure 28: $\quad$ Table - methods for FRF updating.

\section{Further works.}

An integrated methodology for system identification, data comparison and model updating was presented. Many methods used till now only for test data processing are improved and implemented for usage for large FE-Models. Comparison analysis for large data both Test and Analysis were implemented. Combined "simplified" and based on direct updating procedures of validation. Commercial FE-solvers: MSC.Nastran, Permas instead of "own solvers" are used for Model Updating. It is planned to provide in the next time: automatic data processing (modal analysis) for test and analysis models; implementation and development numerous new methods for modal parameters (especially damping) identification and updating. This method can be used in the other branches of industry including in the analysis of the strength calculations of the rail vehicles bodies.

\section{REFERENCES}

[1] EWINS D.: "Modal Testing Theory and Practice", Research Studies Press, 1984

[2] HEYLEN W., LAMMENS S., SAS P. : "Modal Analysis Theory and Testing", Katholieke Universiteit Leuven, Departement Werktuigkunde, Leuven, 1997

[3] GRAFE H.: "Model Updating of large Structural Dynamics Models Using Measured Response Function", PhD. Thesis, Imperial College of Science, Technology and Medicine, Mechanical Engineering, London, UK, 1998

[4] IMAMOVIC N.: "Validation of large structural dynamics models using modal test data", PhD Thesis Imperial College of Science Technology and Medicine, London, UK, 1998
[5] REZA ASHORY M.:"High Quality Modal Testing Methods", PhD Thesis Imperial College of Science Technology and Medicine, London, UK, 1999

[6] VON MACH A.: "Identification of large FEModels", MSC German Users' Conference, 2003

[7] DOMOK S., VON MACH A. ,MERK J.: "FE-OPTIM and MSC/NASTRAN for a Frequency Response Optimization", MSC Americas Users' Conference, 1998

[8] VON MACH A., NOWAK D.: „Balken Pre- und Postprozessor für MSC/NASTRAN feprofilQuerschnitt -Berechnung febeam -Balkensyteme Analyse ", MSC Anwenderkonferenz München 1996

[9] VON MACH A.: "Modal identification of quasisymmetric structures with an example taken from vehicle body models", FENET/NAFEMS Seminar FEM in Structural Dynamics Wiesbaden, 14.-15. 11.2001

[10] ROSE T.: "Using strain energy to identify modal participation in a dynamic response analysis", The MSC World's Users' Conference, Los Angeles, 1994

[11] WAMSLER M., ROSE T.: "Improved identification of contributing modes in superelement modal frequency response analysis", The MSC Americas Users' Conference, Universal City, 1998

[12] WAMSLER M., ROSE T.: "Advanced mode shape identification method for automotive application via modal kinetic energy plots assisted numerous printed outputs", The Americas Users' Conference, Universal City, 1998

[13] BALASUBRAMARIAN B., WAMSLER M.: "Identification of contributing modes in MSC/NASTRAN modal frequency response analysis", MSC European Users' Conference, 1987

[14] KALINSKI K., VON MACH A.: "Modal energy identification of FE large structures", International Conference on Noise and Vibration Engineering ISMA Leuven, 2002, 589-598 
[15] SUTTER T.R., CAMARDA C.J., WALSCH J.L., ADELMAND H.M.: "Comparison of Several Methods for Calculating Vibration Mode Shapes Derivatives", AIAA Journal Vol .26 No.12, 1988

[16] NELSON R.B.: "Simplified Calculation of Eigenvector Derivatives", AIAA Journal, Vol.14, No.9 1976
[17] VON MACH A., UHL T.: "Modal identification of quasi-symmetric structures with an example taken from airplane airframe models", 3rd Worldwide MSC Aerospace Conference Toulose, 2000

[18] FRISWELL M.I., MOTTERSHEAD J.E.: "Finite Element Model Updating in Structural Dynamics", Kluwer Academic Publishers, 1995 